\title{
Treatment of experimental lens capsular tears with intense focused ultrasound
}

\author{
D J COLEMAN,' F L LIZZI, ${ }^{2} \mathrm{~J}$ H TORPEY,' S E P BURGESS,' J DRILLER, \\ A ROSADO, ${ }^{2}$ AND H T NGUYEN'
}

From the 'Cornell University Medical College, Department of Ophthalmology, 1300 York Avenue, New York, New York 10021, and ${ }^{2}$ Riverside Research Institute 330 West 42nd Street, New York, New York 10036

SUMMARY High-intensity focused ultrasound was employed to seal lens capsular tears in a rabbit model. Ultrasound therapy was applied either contiguously, thereby completely covering the tear, or in a discrete exposure pattern around the tear. Both methods prevented the formation of a generalised cataract. This was in contrast to results observed in a group of control (untreated) animals which all developed generalised lens opacities. Each control animal also developed a local lens opacity at the site of the capsular tear, as did half the animals treated with the discrete pattern. No animal treated with contiguous exposures developed any local or generalised traumatic-type cataract other than the small lens opacity immediately produced by the treatment. These treatment cataracts would not constitute a significant impediment to vision so long as they did not fall on the visual axis.

Mechanical injury to the human lens capsule usually results in the formation of a lens opacity at the site of damage. This local cataract typically progresses to complete opacification of the lens. Rarely the lens capsule may heal, leaving an opacity that remains confined to the region of the capsular rupture. Early removal of the lens is accepted therapy for such an injury, since retrospective studies have shown that its late removal has a higher morbidity.$^{1-3}$ Fibrin derivatives have been used as a tissue 'adhesive' to repair lens capsule perforations with some success. ${ }^{45} \mathrm{We}$ have explored a different approach using intense focused ultrasound (IFU). We now have considerable experience with ultrasound-induced lesions in the eye, both in humans and in animals. In this department we have used IFU to treat a variety of diseases including glaucoma, tumours, and vitreous membranes. ${ }^{6-8}$ We have found that with proper care and a well designed system the technique is easy to use and has a low incidence of side effects. It also has the advantage of being non-invasive and can be performed outside the operating room.

A number of investigators ${ }^{9-11}$ have studied cataracts produced with high-intensity ultrasound. These cataracts remained stable with time and did not progress to generalised opacification of the lens. The

Correspondence to D Jackson Coleman, MD. aim of this study was to evaluate whether a lens capsular rupture could be sealed with IFU and thereby prevent the formation of a generalised cataract. If successful this type of treatment might be beneficial either alone or in combination with fibrin glue techniques. ${ }^{45}$ It might also prove useful as a palliative measure to reduce inflammation from exposed lens material.

\section{Material and methods}

Thirteen Dutchbelt rabbits were anaesthetised (Ketamine $100 \mathrm{mg}$ and xylazine $15 \mathrm{mg}$ intramuscularly). One pupil was dilated with phenylephrine hydrochloride $(10 \%)$ and the cornea anaesthetised locally with proparacaine hydrochloride $(0 \cdot 5 \%)$. A 30 gauge needle, introduced through the limbus into the anterior chamber, was used to puncture and then to tear the anterior lens capsule. This manoeuvre produced either a narrow $4 \mathrm{~mm}$ anterior capsular tear or a gaping lesion up to $4 \mathrm{~mm}$ in diameter (Fig. 1).

Five rabbits served as controls. The lens of one eye of each animal was prepared as above but received no further treatment. The lenses were examined and photographed twice weekly over a 90-day period.

Eight rabbits received ultrasonic treatment approximately 30 minutes after capsular injury. The anaesthetised rabbits were prepared for immersion 


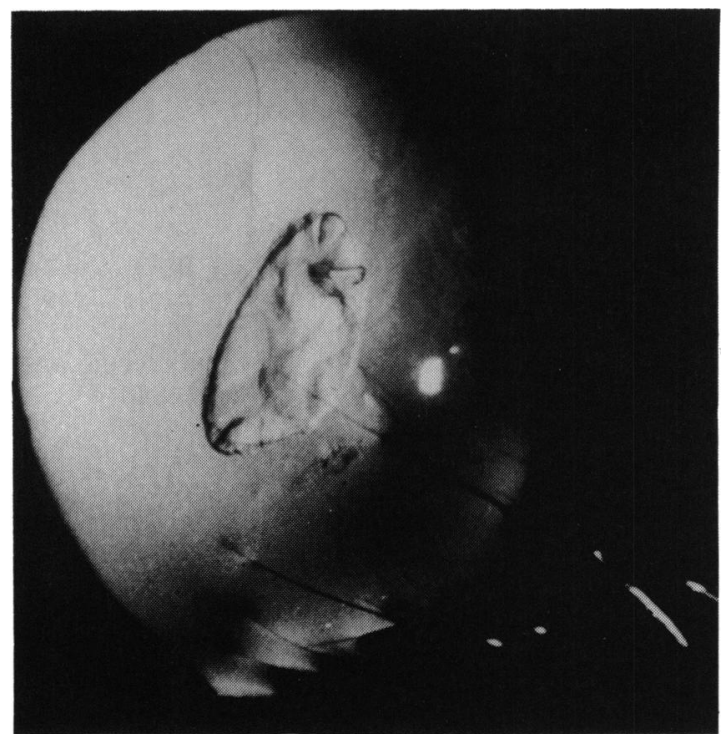

Fig. 1 Anterior capsular tear immediately after injury. ultrasonic therapy in a manner previously described. ${ }^{12}$ The ultrasound transducer was then inserted into the coupling saline solution bathing the globe, and multiple small cataracts were induced in the lens so as completely to cover the laceration. The extent of the lesions could be assessed from the opacified region of the lens. In one series of treatments (contiguous type) the lesions did overlap, but in the second series (discrete type) the lesions did not overlap (Fig. 2). Since each capsular tear varied in size and shape, 1245 ultrasonic lens opacities were required to cover the tear. After treatment the animals were examined periodically over 90 days.

The transducer assembly ${ }^{13}$ was a triaxial unit which held a therapeutic transducer with a central aperture. A diagnostic transducer was located within the therapeutic transducer aperture. A central aiming light was used for azimuthal positioning. The diagnostic transducer was connected to a standard diagnostic Ascan unit (Sonometrics Ophthalmoscan) and was used to ensure that the centre of the focal zone of the therapeutic beam was located on the anterior surface

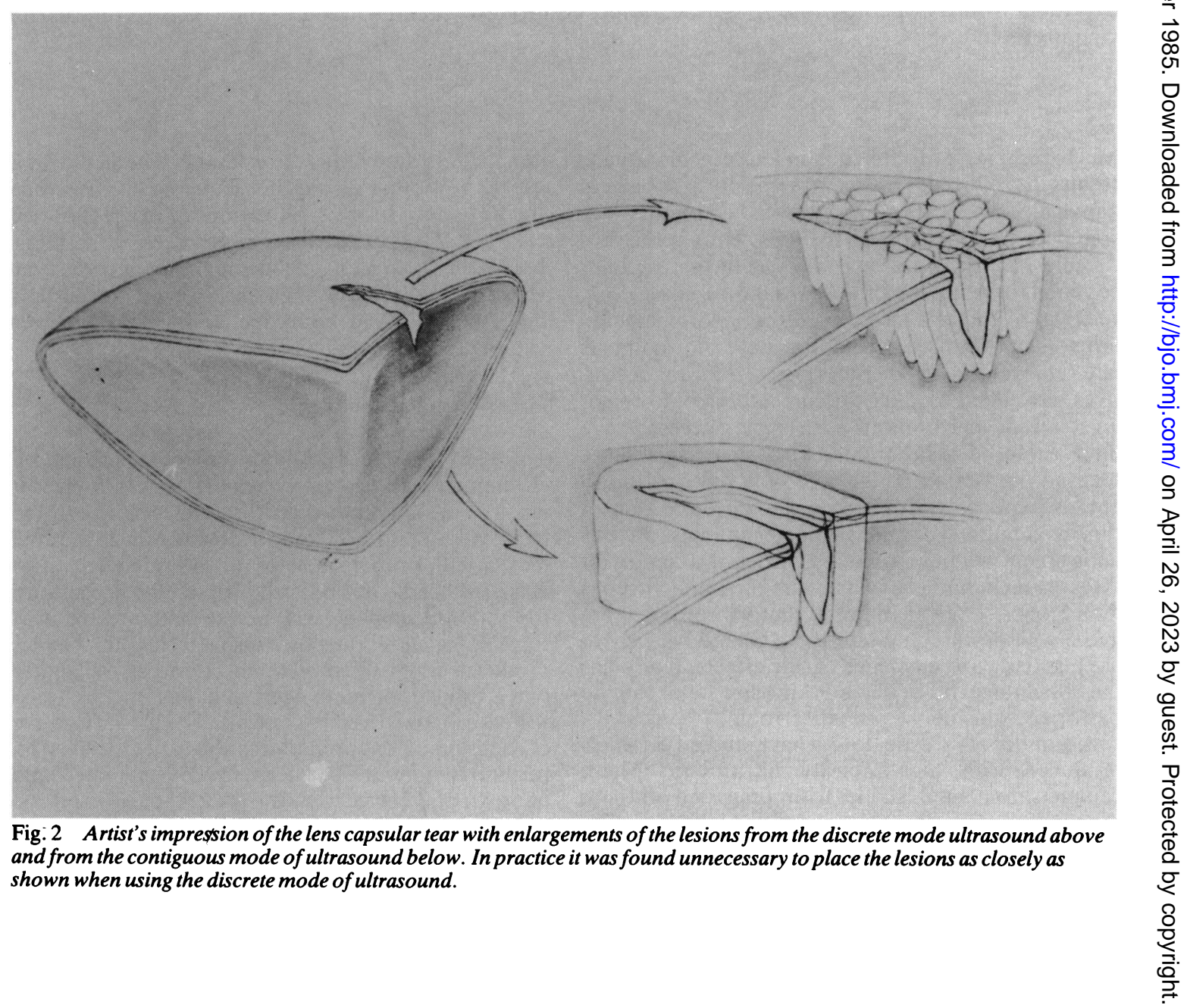



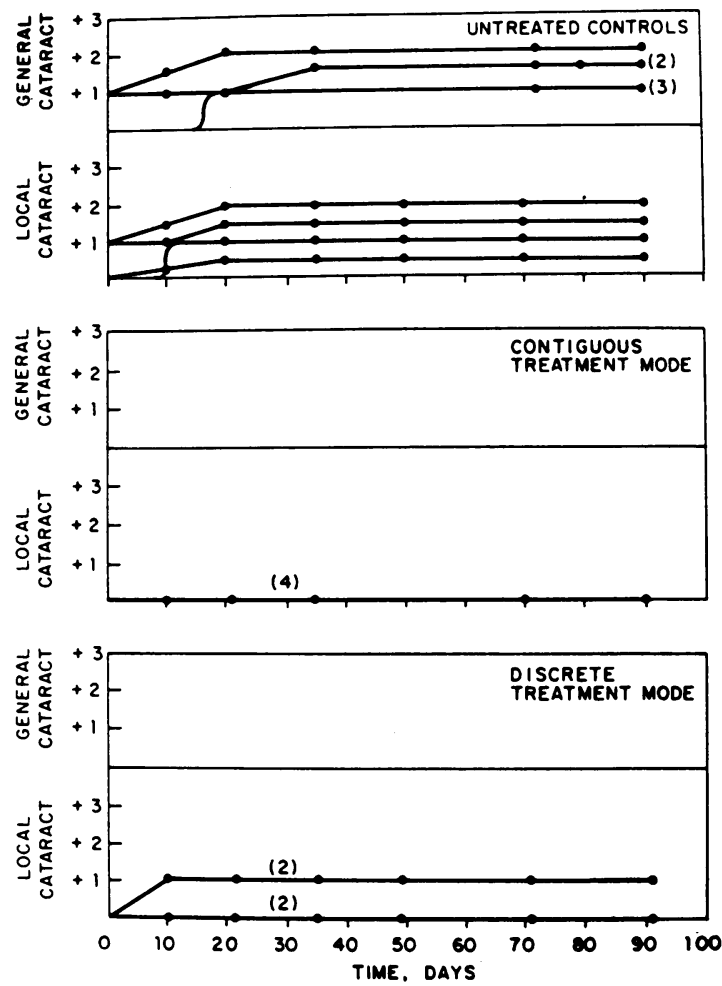

Fig. 3 Extent and severity of lens opacities in both treatment groups and the control group and also the progression of these opacities with time.

of the lens (axial positioning). The resonant frequency of transducer was $1.5 \mathrm{MHz}$ and in these studies it was operated at its third harmonic $(4.75 \mathrm{MHz})$. At the third harmonic the half-power axial depth of focus is $3 \mathrm{~mm}$ and the half-power transverse width of the focal zone is $0.37 \mathrm{~mm}$. In this report spatial average temporal peak (SATP) intensities are cited which refer to the SATP values within the focal plane at half-power beam width.

The lens opacities from the discrete type of treatment, which formed a row of non-overlapping lesions, were each produced with a $20 \mathrm{~ms}$ pulse at an intensity of $2000 \mathrm{~W} / \mathrm{cm}^{2}$ (SATP). Under the contiguous type of treatment the lens opacities, which form a complete blanket of lesions, were formed with 20 ms pulses generated at 10 pulses per second at the same intensity level.

\section{Results}

All cataracts resulting from the capsular injury were categorised as 'local' or 'general' according to whether they were localised to the tear or had spread into the entire lens. They were also graded as to degree (slight

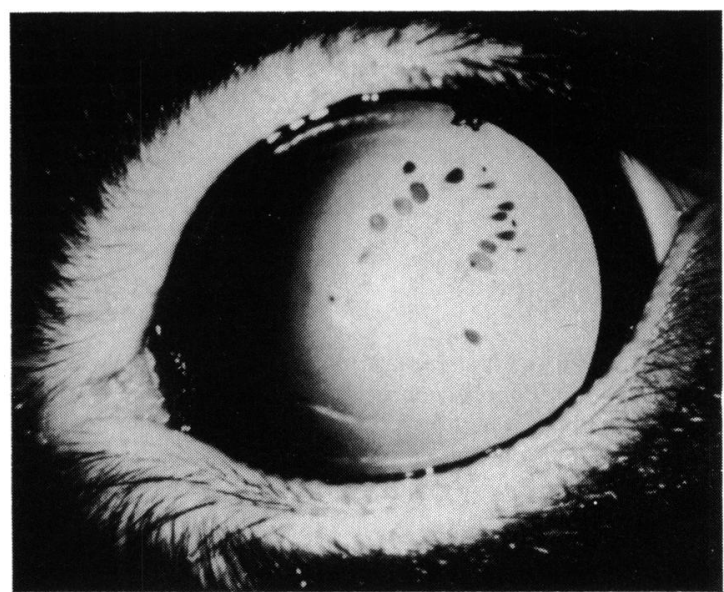

Fig. 4a Lens treated by the discrete mode, showing individual ultrasonically induced lens opacities in an otherwise clear lens 90 days after treatment.

$(+1)$, moderate $(+2)$, severe $(+3))$. Although these gradations are not quantitative, they did allow qualitative comparison of various lesions over the 90day period.

Fig. 3 shows the results for the untreated control group, the group receiving the contiguous mode of therapy, and the group receiving the discrete mode of therapy. The incidence of generalised and localised cataract is shown for each treatment group together with a qualitative estimation of its degree and time relationship to the lens capsular rupture. It is seen

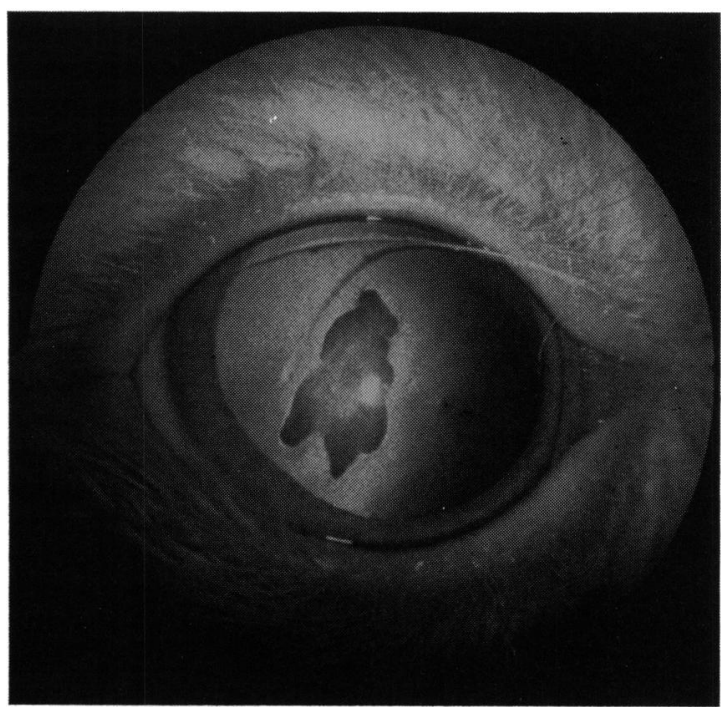

Fig. 4b Lens treated by the contiguous mode, showing single ultrasonically induced lens opacity 90 days after treatment. 
Fig. 5 Large, discrete, irregular shaped area of homogeneous pale pink material within the ultrasonically induced lens opacity. (Haematoxylin and eosin, $\times 18$ ).

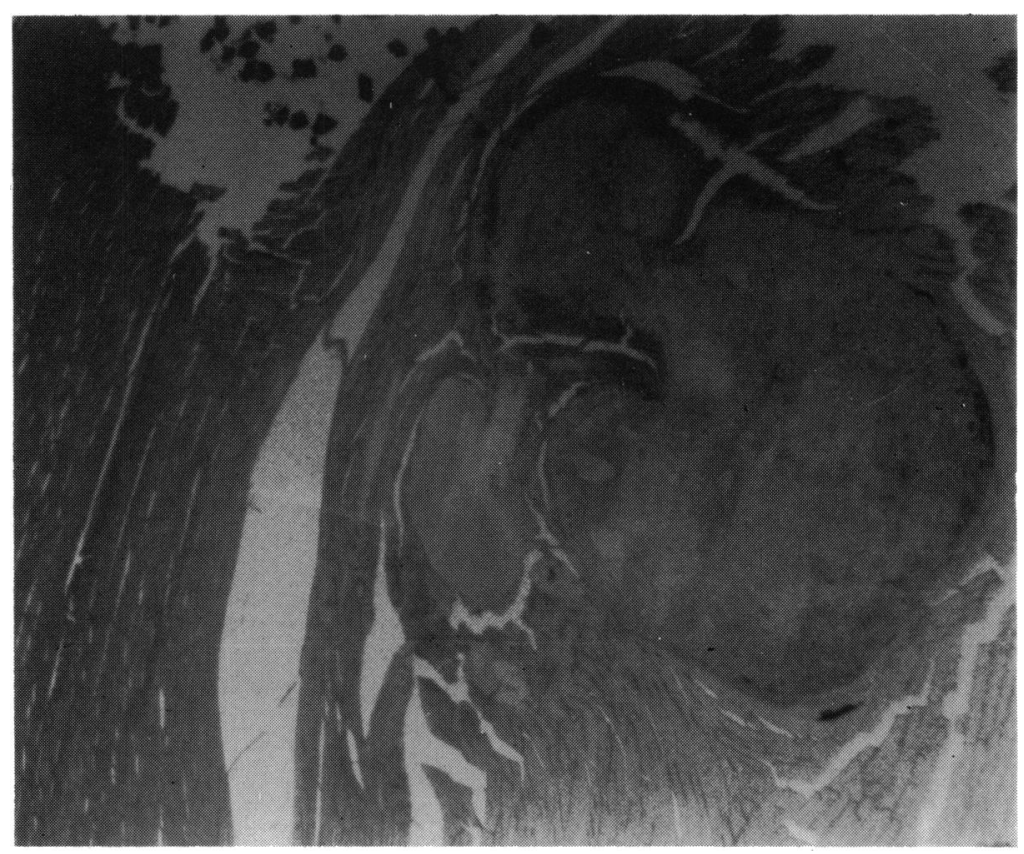

that all untreated lenses developed mild generalised cataract within three weeks. All lesions that received the contiguous mode of treatment had no local or general extensions. Two of the four lenses that received the discrete mode of treatment showed limited local extension, which stabilised after about 10 days. None of the lenses receiving the discrete form of treatment showed any general extension of the cataract induced by the capsular tear (Figs. 4a, b). In addition none of the ultrasonically induced cataracts grew in size over the study period.

Light microscopic examination of a lens treated with the contiguous mode showed the following features. In the inner cortical region the lens fibres

Fig. 6 Photomicrograph: Small globular structures and granular brown pigment within the ultrasonically induced lens opacity. (Haematoxylin and eosin, $\times 180$ ).

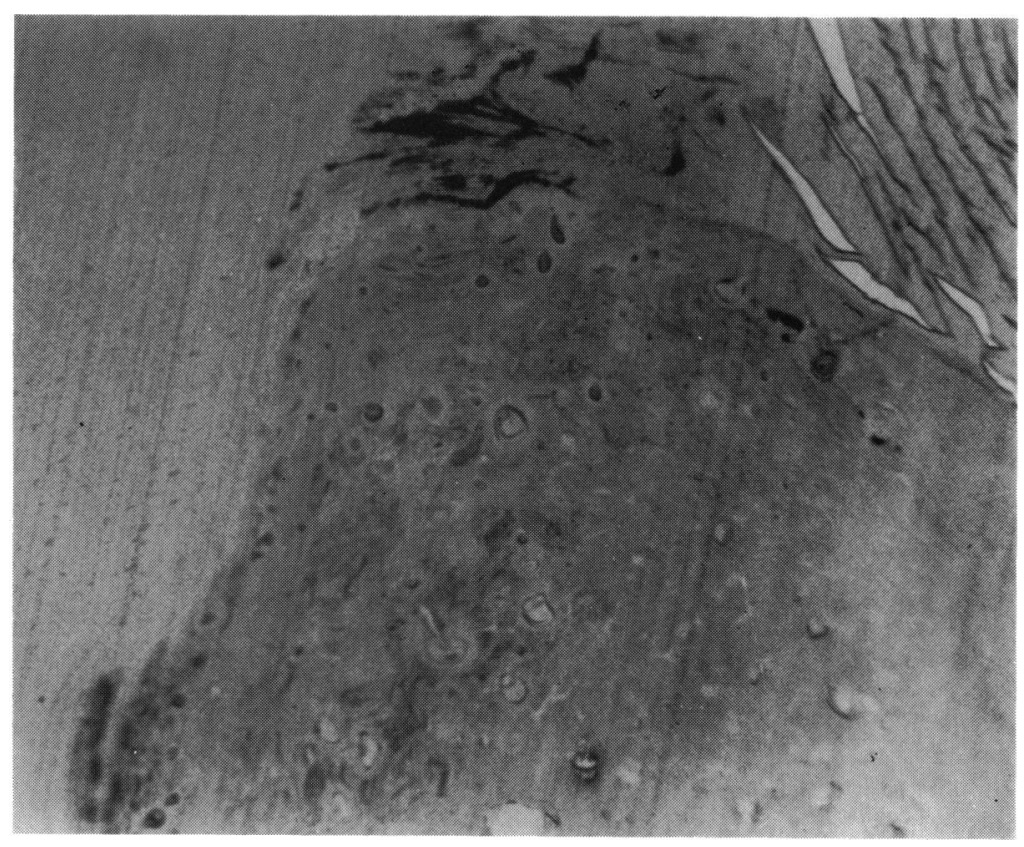


were interrupted by large, multiple, discrete, irregular shaped areas of homogeneous pale pink appearance (Fig. 5). At the periphery of these homogeneous areas small globular structures of varying sizes were frequently seen. Within the homogeneous areas granular brown pigment was occasionally observed (Fig. 6). The lens fibres immediately adjacent to the homogeneous areas occasionally showed minimum disorganisation. The remaining lens fibres outside the treated areas appeared normal.

In a lens treated by the discrete method lenticular changes were similar to those noted above except that the lesion was smaller and more sharply delineated. It was also situated in the inner cortical zone. The lesion in this lens contained a larger amount of granular brown pigment than the other lens.

\section{Discussion}

In this animal study we have demonstrated that the development of a generalised traumatic cataract following capsular rupture can be prevented by the use of a matrix of ultrasonically induced lens opacities that are small, localised, and stable. This therapy could be used in the management of lens injury in humans. The usual treatment for a lens with a medium or large capsular rupture is its rapid removal, since generalised cataract develops very frequently. However, small through-and-through intraocular injuries due to foreign bodies may heal without cataract formation, as may small peripheral tears. Early removal of the lens is technically easier and associated with less uveitis and more rapid visual rehabilitation. ${ }^{214}$ However, there are major problems associated with monocular aphakia, particularly in the very young. If an otherwise clear lens with a damaged capsule could be saved, this would offer a considerable advantage for preserving useful binocular vision. Eagling ${ }^{15}$ found that, in seven out of eight patients under the age of 8 years with a penetrating injury involving only the lens and cornea, the visual acuity was less than $6 / 18$ at 18 months. In comparison $65 \%$ of a group of all patients with similar injuries achieved a visual acuity of $6 / 12$ or better. In addition 12 of her 20 patients successfully fitted with contact lenses complained of diplopia. ${ }^{15}$

This method of ultrasonically sealing lens capsular tears might allow retention of an injured lens if the following conditions are met. Firstly, the capsular damage must be outside the visual axis. Secondly, the time lapse between injury and ultrasonic sealing must be relatively short, probably less than 24 hours. Thirdly, the safety of the procedure needs further evaluation, particularly in respect of whether ultrasound application results in increased technical difficulties with subsequent lens removal, should this prove necessary. Further studies are needed to delineate the operative mechanism responsible for 'sealing' the lens capsular tear, to identify optimal exposure levels, and to study the long-term benefits of this procedure. The initial results are promising, and together with other advantages of ultrasonic therapy they offer prospects of a practical, easy-touse technique.

This work was supported in part by NIH Grants EY03174 (D J Coleman) and EY01480 (F I Lizzi), the Dyson Foundation, and Research to Prevent Blindness, Inc.

\section{References}

1 Muga R, Maul E. The management of lens damage in perforating corneal lacerations. Br J Ophthalmol 1978; 62: 784-7.

2 Kearns WP. A retrospective evaluation of the traumatic cataract. Ann Ophthalmol 1977; 9: 1271-4.

3 Perlman EM, Albert DM. Clinically unsuspected phacoanaphylaxis after ocular trauma. Arch Ophthalmol 1977; 95: 244-6.

4 Buschchmann W, Waller W, Gehrig V, Vogt E. Experimental studies on the treatment of lens capsule perforations. Abstracts of the 20th meeting of the Association for Eye Research: Ophthalmic Res 1980; 12: 146.

5 Riebel $\mathrm{O}$. Extraction of magnetic foreign bodies from the clear lens. Am J Ophthalmol 1979; 88: 935-8.

6 Coleman DI, Lizzi FL, Driller J, et al. Therapeutic ultrasound in the treatment of glaucoma. Part II: Clinical applications. Ophthalmology (Rochester) 1985; 92: 347-53.

7 Lizzi FL, Coleman DJ, Driller J, Franzen LA, Jakobiec FA. Experimental, ultrasonically induced lesions in the retina, choroid and sclera. Invest Ophthalmol Vis Sci 1978; 17: 350-60.

8 Coleman DJ, Lizzi FL, Jakobiec FA. Therapeutic ultrasound in the production of ocular lesions. Am J Ophthalmol 1978; 86: 185-92.

9 Lavine O, Langenstrass K, Bower C, Fox F, Griffing V, Thaler W. Effect of ultrasonic waves of the refractive media of the eye. Arch Ophthalmol 1952; 47: 204-19.

10 Torchia RT, Purnell EW, Sokullu A. Cataract production by ultrasound. Am J Ophthalmol 1967; 64: 305-9.

11 Coleman DJ, Lizzi FL, Burt WJ, Wen H. Properties observed in cataracts produced experimentally with ultrasound. Am J Ophthalmol 1971; 71: 1284-8.

12 Lizzi FL, Packer AJ, Coleman DJ. Experimental cataract production by high frequency ultrasound. Ann Ophthalmol 1978; 10: 934-42.

13 Coleman DJ, Lizzi FL, Chang S, Driller J. Applications of therapeutic ultrasound in ophthalmology, Prog Med Ultrasound 1981; 2: 263-70.

14 Jain IS, Bansal SL, Dhir SP, Kaul RL, Gangwar MS. Prognosis in traumatic cataract surgery. J Pediatr Ophthalmol 1979; 16: 301-5.

15 Eagling EM. Perforating injuries of the eye. Br J Ophthalmol 1976; 60: 732-6. 